\title{
THE
}

\section{Clinical Implications of Vancomycin Heteroresistant and Intermediately Susceptible Staphylococcus aureus}

\author{
Diane M. Gomes \\ University of Rhode Island, diane_gomes@uri.edu \\ E. Ward \\ University of Rhode Island, kward@uri.edu \\ Kerry L. LaPlante \\ University of Rhode Island, kerrylaplante@uri.edu
}

Follow this and additional works at: https://digitalcommons.uri.edu/php_facpubs

The University of Rhode Island Faculty have made this article openly available.
Please let us know how Open Access to this research benefits you.

This is a pre-publication author manuscript of the final, published article.

Terms of Use

This article is made available under the terms and conditions applicable towards Open Access Policy Articles, as set forth in our Terms of Use.

\section{Citation/Publisher Attribution}

Gomes, D. M., Ward, K. E., \& LaPlante, K. L. (2015). Clinical Implications of Vancomycin Heteroresistant and Intermediately Susceptible Staphylococcus aureus. Pharmacotherapy, 35(4), 424-432. doi: 10.1002/ phar.1577

Available at: http://dx.doi.org/10.1002/phar.1577 


\section{REVIEW ARTICLE}

Clinical implications of vancomycin heteroresistant and intermediately susceptible Staphylococcus aureus

Diane M. Gomes, ${ }^{1,3}$ Kristina E. Ward, ${ }^{1}$ and Kerry L. LaPlante ${ }^{1,2,3^{*}}$

University of Rhode Island, Department of Pharmacy Practice ${ }^{1}$, Warren Alpert Medical School of Brown University ${ }^{2}$, and Veterans Affairs Medical Center, Providence, $\mathrm{RI}^{3}$

*Corresponding Author: Kerry L. LaPlante, Associate Professor of Pharmacy, University of Rhode Island, 7 Greenhouse Road, Suite 295A, Kingston, RI 02881, Office: (401) 874-5560 ; Fax: (401) 457-3305; E-mail: KerryLaPlante@uri.edu

Keywords: Heterogeneous Vancomycin-Intermediate Staphylococcus aureus, VancomycinIntermediate Staphylococcus aureus, Methicillin-Resistant Staphylococcus aureus, minimum inhibitory concentration, resistance, intermediate, screening, Staphylococcus aureus, reduced susceptibility, vancomycin

Financial Support: None

Running Head: Vancomycin reduced susceptibility in Staphylococcus aureus

Manuscript Word Count: 3699 
1 Abstract. Staphylococcus aureus has proven to be a major pathogen with the emergence of

2 methicillin-resistant S. aureus (MRSA) infections and recently with heteroresistant vancomycin

3 intermediate S. aureus (hVISA) and vancomycin intermediate S. aureus (VISA) infections. While

4 vancomycin is traditionally a first line and relatively effective antibiotic, its continued use is under

5 question, as reports of heteroresistance in S. aureus isolates are increasing. Both hVISA and VISA

6 infections are associated with complicated clinical courses and treatment failures. The prevalence,

7 mechanism of resistance, clinical significance, and laboratory detection of hVISA and VISA

8 infections are not conclusive, making it difficult to apply research findings to clinical situations.

9 We provide an evidence based review of S. aureus isolates expressing heterogenic and reduced 10 susceptibility to vancomycin. 


\section{Introduction}

12 Methicillin-resistant Staphylococcus aureus (MRSA) is the most commonly encountered bacteria

13 in hospitals and community settings ${ }^{1}$ and is associated with invasive infections ranging in severity

14 from mild to fatal. ${ }^{2}$ Vancomycin is considered the standard treatment for empiric and definitive

15 serious MRSA infections. ${ }^{2}$ In recent years, infections caused by MRSA with reduced

16 susceptibility to vancomycin have emerged. The formation of intermediate resistant isolates is

17 likely caused by selection pressure from ever-present and longstanding use of vancomycin. ${ }^{3-5}$ Poor

18 patient outcomes are attributed to heteroresistant vancomycin intermediate S. aureus (hVISA) and

19 vancomycin intermediate $S$. aureus (VISA) infections. ${ }^{6-8}$ Herein we review the prevalence,

20 laboratory detection and interpretation, resistance mechanisms, risk factors and outcomes,

21 treatment options, and infection control strategies for hVISA and VISA. Peer-reviewed

22 publications were identified using PubMed, Embase, and Cochrane Central Register of Controlled

23 Trials.

\section{Prevalence of hVISA and VISA}

26 The first clinical strain of $S$. aureus with intermediate resistance to vancomycin, designated Mu50,

27 was reported in 1997 from Japan..$^{9,10}$ The first hVISA isolate, designated Mu3, was identified in

28 Japan one year earlier from a patient with MRSA pneumonia unresponsive to vancomycin. ${ }^{9}$ Since

29 then, hVISA and VISA cases have been reported in the United States, United Kingdom, China,

30 Australia, Turkey, France, Belgium, Germany, Italy, Brazil, and South Korea. ${ }^{11}$ The true

31 prevalence of hVISA is unknown, and estimates vary widely because of non-standardized

32 detection methodologies or absence of routine hVISA screening, variation in interpretation, 
33 geographical location, clinical setting, and differing patient populations. ${ }^{12-19}$ Reported rates of

34 hVISA throughout the world range from 0 to $73.7 \% .^{18}$

36 One retrospective study evaluated MRSA strains with heterogenic intermediate resistance to

37 vancomycin over a 22-year period in three Detroit hospitals. The prevalence of these organisms

38 increased from $2.2 \%(1986-1993)$ and $7.6 \%(1992-2002)$ to $8.3 \%$ between 2003 and $2007 .{ }^{16}$

39 Only 14 of the $1,498(0.93 \%)$ MRSA isolates were identified as VISA. There was no apparent

40 pattern of increasing prevalence over the three time periods for VISA isolates. An increase in

41 hVISA was also described in a similar retrospective study from Turkey of $1.6 \%$ in 1998 to $36 \%$

42 in $2001 .^{20}$ Because clonality was not evaluated in either study, the increase in prevalence may

43 have reflected clonal spread rather than true prevalence. Prevalence may have been

44 underestimated because the isolates were stored for prolonged periods in glycopeptide-free media,

45 which may result in a loss of resistance. ${ }^{21}$ Two surveillance studies conducted in 2009 and 2011

46 in over 40 U.S. medical centers determined rates of antimicrobial resistance among S. aureus

47 isolates collected from patients with infections. ${ }^{22,23}$ The rates of hVISA among MRSA isolates in

482011 were higher than in $2009(1.2 \%$ vs. $0.4 \%, \mathrm{P}=0.003) .{ }^{22}$ Of note, strains of VISA were not

49 detected. ${ }^{22,23}$ While the current prevalence of VISA is low, these organisms may become more

50 common in the future. Data suggests that heteroresistance is a precursor to VISA, therefore the

51 suspected increase in prevalence of hVISA may predict more VISA infections. Increased use of

52 vancomycin provides selection pressure for further emergence of VISA. Based on available data,

53 hVISA appears to be on the rise, yet VISA still remains a rare occurrence. Additional studies are

54 needed to determine appropriate surveillance methods because retrospective studies are 
55 complicated by the ability of hVISA to revert back to vancomycin-susceptible S. aureus (VSSA)

56 and VISA to revert back to hVISA.

58 hVISA and VISA Laboratory Detection and Interpretation

59 Further discussion of hVISA and VISA require that clinical and microbiologic definitions are

60 addressed. In 2006, the Clinical and Laboratory Standards Institute (CLSI) lowered vancomycin

61 minimum inhibitory concentration (MIC) breakpoints for S. aureus. ${ }^{24}$ The CLSI breakpoints by

62 broth microdilution (BMD) currently define vancomycin susceptibility as an $\mathrm{MIC} \leq 2 \mu \mathrm{g} / \mathrm{mL}$,

63 vancomycin-intermediate susceptibility as an MIC of 4 to $8 \mu \mathrm{g} / \mathrm{mL}$, and vancomycin resistance as

64 an MIC of $\geq 16 \mu \mathrm{g} / \mathrm{mL}$ (Table 1). ${ }^{25}$ Vancomycin MIC breakpoints were lowered in an effort to

65 increase detection of potentially heterogeneous-intermediate isolates because of reported

66 associations between vancomycin treatment failure and S. aureus isolates with MICs $\geq 4$

$67 \mu \mathrm{g} / \mathrm{mL} .^{7,8,25}$ Heteroresistance refers to the presence of less susceptible subsets within a larger

68 population of fully antimicrobial-susceptible microorganisms. ${ }^{5}$ When tested using routine

69 methods, hVISA isolates are susceptible to vancomycin (MIC $\leq 2 \mu \mathrm{g} / \mathrm{mL}$ ) but contain

70 subpopulations that express reduced vancomycin susceptibility (MIC $\geq 4 \mu \mathrm{g} / \mathrm{mL}){ }^{11}$

72 Detection of hVISA is a great challenge in clinical microbiology laboratories because reliable and

73 practical methods are not currently available for routine use. Heteroresistant subpopulations are

74 present in low frequencies $\left(\geq 1 \times 10^{6}\right)$ and can grow in higher vancomycin concentrations than the

75 MIC predicts. Such small populations may not be detected by the inocula $\left(5 \times 10^{5} \mathrm{CFU} / \mathrm{mL}\right)$

76 used in standard CLSI microbiology methods. As a result, hVISA isolates are likely undetected

77 in clinical laboratories that use traditional MIC testing methodology. ${ }^{13}$ Population analysis 
78 profiling with area under the curve (PAP-AUC) is the current reference standard method for

79 confirming hVISA and is the most reliable and reproducible test. However PAP-AUC is labor-

80 intensive, time consuming (3 to 5 days), and costly for use in clinical microbiology

81 laboratories. ${ }^{17,19,26}$ Consequently, several screening methods have been developed, such as

82 glycopeptide resistance detection (GRD), marcromethod E-test (MET) and brain heart infusion

83 (BHI) screen agar plates (Table 2). ${ }^{27-29}$ However, none of these tests have the same degree of

84 sensitivity and specificity as the PAP-AUC test, with issues of reproducibility and variability, in

85 reporting results. ${ }^{19}$ Until a suitable hVISA detection method becomes available for use in clinical

86 microbiology laboratories, routine testing is not currently recommended. ${ }^{2}$ Currently, clinical

87 screening for hVISA isolates in high-risk patients is favored (Table 3), particularly in patients who

88 do not respond to vancomycin. Further research is warranted to develop a detection method that

89 is practical, cost-effective, and reliable for routine use in clinical settings.

90

91 Non-automated MIC methods for the detection of VISA are recommended by the Centers for

92 Disease Prevention and Control (CDC). ${ }^{30}$ Acceptable non-automated MIC methods for detecting

93 VISA include BMD per CLSI, agar dilution, and Etest (0.5 McFarland). ${ }^{30}$ Though automated

94 methods and vancomycin screen agar plates can be useful in the detection of VISA isolates with a

95 vancomycin MIC of $8 \mu \mathrm{g} / \mathrm{mL}$, sensitivity levels have not been determined for $S$. aureus with

96 vancomycin MICs of $4 \mu \mathrm{g} / \mathrm{mL}^{30}$ In these situations, a second method, such as BMD per CLSI

97 criteria, should be used to confirm VISA isolates. ${ }^{30}$

98

99 Current susceptibility testing methods do not consistently distinguish between MICs of 1 and 2 $100 \mu \mathrm{g} / \mathrm{mL}^{2,31}$ Therefore, laboratory results should indicate the methodology used, because 
101 vancomycin MIC results will differ between methods and may alter treatment decisions. ${ }^{11}$ In

102 comparison to the CLSI BMD method, automated detection methods, particularly Phoenix system

103 and Vitek, tend to underestimate the MIC, while E-test and MicroScan (prompt method) may

104 overestimate the MIC. ${ }^{31}$ Precision of these methods is clinically important as higher vancomycin

$105 \operatorname{MICs}(>1.5 \mu \mathrm{g} / \mathrm{mL})$ are associated with poorer outcomes (e.g., increased mortality, recurrence,

106 delayed response, treatment failure, prolonged hospitalization), particularly in high inoculum

107 infections and with a higher proportion of hVISA presence. ${ }^{25,32}$ Alternative therapies should be

108 considered for patients receiving vancomycin therapy who are persistently bacteremic ( $\geq 7$ days)

109 or who have no clinical improvement despite source control with an MIC of $\geq 1.5 \mu \mathrm{g} / \mathrm{mL}$ by

110 Etest. ${ }^{2,31,32}$

111

\section{Resistance Mechanisms of hVISA and VISA}

113 Evidence suggests that hVISA and VISA arise during continued or sub-optimal exposure to

114 vancomycin. ${ }^{7,33}$ The proposed mechanism is selective pressure by vancomycin resulting in the

115 development of rare vancomycin-resistant clones that progress to hVISA and, with continued-

116 exposure, to a uniform population of VISA clones. ${ }^{5,9}$ These isolates have significant differences

117 in cell physiology, including morphologic changes and genetic alterations. Strains of hVISA and

118 VISA are characterized by thicker cell walls that correlate with increased vancomycin MICs. ${ }^{34}$

119 Cell wall thickening impairs intracellular penetration of vancomycin rendering it ineffective. . $^{5,34}$

120 In addition, hVISA and VISA are associated with slower growth rates than fully susceptible

121 strains, which may contribute to persistent and recurrent infections. ${ }^{35}$ Other mechanisms of

122 resistance include alterations in transcriptional and metabolic genes and loss-of-function mutations

123 that disturb critical cell wall biosynthesis. ${ }^{11}$ The accessory gene regulator (agr) operon directs 
124 many critical virulence pathways, particularly the production of exotoxins. ${ }^{11}$ In hVISA and VISA

125 strains, agr function is reduced, favoring the development of vancomycin resistance and

126 potentially promoting biofilm production that ultimately enhances the survival of hVISA and

127 VISA. ${ }^{33,36,37}$

129 Risk Factors and Outcomes Associated with hVISA and VISA

130 Heteroresistance has been reported in MRSA isolates with MICs as low as $0.5 \mu \mathrm{g} / \mathrm{mL}$ and in cases

131 where vancomycin was minimally effective. ${ }^{6,16}$ Several studies have noted an increase in

132 vancomycin treatment failures and mortality with vancomycin susceptible MRSA strains,

133 particularly those with MICs of 1.5 or $2 \mu \mathrm{g} / \mathrm{mL} \cdot{ }^{25,32,38-40}$ A recent meta-analysis of 20 studies

134 evaluated high versus low vancomycin MICs $(\geq 1.5 \mu \mathrm{g} / \mathrm{mL}$ vs $<1.5 \mu \mathrm{g} / \mathrm{mL}$, respectively) on

135 clinical outcomes in adults with MRSA infections. ${ }^{40}$ An increased risk of failure was observed in

136 the high MIC group compared to the low MIC group (relative risk [RR], 1.40; 95\% confidence

137 interval $[\mathrm{CI}], 1.15-1.71)$. There was also a greater risk of overall mortality (RR, $1.45 ; 95 \%$

138 CI,1.08-1.87) in the high MIC group. Although the investigators attempted to exclude hVISA

139 isolates, hVISA presence was not tested in every study, which may have contributed to

140 vancomycin treatment responses. While most of the isolates were from blood, clinical

141 heterogeneity cannot be excluded. Another study evaluated 559 MRSA isolates and found an

142 increased incidence of hVISA when the vancomycin MIC shifted from 1 to $2 \mu \mathrm{g} / \mathrm{mL}$. $^{41}$ The

143 incidence of hVISA was nearly $40 \%$ in isolates with an MIC of $2 \mu \mathrm{g} / \mathrm{mL}$, supporting the results of

144 other studies that suggest the proportion of hVISA isolates are directly related to increases in

145 vancomycin MIC. 6,15,23,41 Increases in vancomycin MICs are hospital specific and perhaps caused

146 by clonal outbreaks. However, this highlights the trends of vancomycin tolerance, which may be 
147 caused by overuse of vancomycin, sub-therapeutic vancomycin concentrations, high bacterial load, 148 or slow vancomycin bactericidal activity. 3,42

150 Both hVISA and VISA have been identified in hospital and community strains of MRSA and in 151 MSSA. ${ }^{16}$ The findings of studies that evaluated clinical predictors and outcomes of hVISA 152 infections are inconsistent. This may be attributed to the considerable heterogeneity of these 153 studies, including differences in study design, clinical definitions, selection of isolates (initial 154 isolate, final isolate, or random selection), patient populations, and testing methodologies. 155 Commonly reported associations with hVISA infections include vancomycin treatment failure and 156 high-inoculum MRSA infections (e.g., bacteremia, infective endocarditis, osteomyelitis, deep 157 abscesses, and prosthetic device infections). ${ }^{6,7,14,33,43,44}$ Other potential predictors of hVISA and 158 VISA infections are prior MRSA infection or colonization (previous 3 months), previous 159 vancomycin exposure (prior 6 months), initial low serum vancomycin trough levels $(<10 \mu \mathrm{g} / \mathrm{mL})$, 160 persistent bacteremia ( $\geq 7$ days), and presence of indwelling devices (Table 2). ${ }^{7,8,12,14,44,45} 46$

162 Patients with hVISA infections tend to experience prolonged clinical courses, suboptimal response 163 to vancomycin therapy, and prolonged hospital stays. ${ }^{6-8,14,33,42,44}$ One retrospective case-control 164 study compared the clinical features and outcomes of hVISA bacteremia $(\mathrm{n}=27)$ and MRSA 165 bacteremia $(n=223) .{ }^{14}$ Compared with MRSA bacteremia, patients with hVISA infections had 166 significantly more days of bacteremia (median duration, 12 days vs. 2 days, respectively; $\mathrm{P}=$ $1670.005)$ and significantly higher rates of endocarditis $(18.5 \%$ vs. $3.6 \%$, respectively; $\mathrm{P}=0.007)$ and 168 osteomyelitis $(25.9 \%$ vs. $7.2 \%$, respectively; $\mathrm{P}=0.006) .{ }^{14}$ Of note, patients in the hVISA group 169 had significantly more prosthetic/implant devices (e.g., artificial heart valves, pacemakers, or 
170 orthopedic implants) and surgical site infections (in the previous month) at baseline, which may

171 have attributed to poorer outcomes. In a small case series, glycopeptide treatment failure, (defined

172 as a positive $S$. aureus blood culture after $\geq 7$ days of glycopeptide therapy or a sterile site culture

173 positive for $S$. aureus after $\geq 21$ days of glycopeptide therapy) occurred in 19 of $25(76 \%)$ patients

174 with hVISA infections (bacteremia, endocarditis, osteomyelitis, or septic arthritis). ${ }^{8}$

175

176 A retrospective, multicenter, matched cohort study compared the outcomes of hVISA versus

177 vancomycin susceptible-MRSA (VS-MRSA) bloodstream infections (BSI) and found similar

178 results. $^{6}$ Study investigators concluded that rates of vancomycin treatment failure were 11 times

179 higher for a patient with hVISA BSI (50/61, 82\%) than VS-MRSA BSI $(20 / 61,32.8 \%$; P <0.001).

180 Patients with hVISA BSI were also more likely than patients with VS-MRSA BSI to have 181 persistent bacteremia (59\% vs. $21.3 \%$, respectively; $\mathrm{P}<0.001)$, infection recurrence at 60 days 182 (25.5\% vs. $1.9 \%$, respectively; $\mathrm{P}<0.001)$, and longer hospital length of stay (median in days, 24

183 vs. 16 , respectively; $\mathrm{P}=0.022$ ). While differences in 30-day MRSA infection-related mortality 184 and all-cause 30-day mortality were not observed between the hVISA BSI group and VS-MRSA 185 BSI group (21.3\% vs. $9.8 \% ; \mathrm{P}=0.081$ and $24.6 \%$ vs. $11.5 \% ; \mathrm{P}=0.076$, respectively). Similarly, 186 no other studies have been powered to detect a significant difference in mortality between hVISA 187 and non-hVISA infections. A recent systematic review and meta-analysis evaluated 30-day 188 mortality from eight comparative hVISA studies. ${ }^{18}$ After combining the data, 30-day mortality 189 between hVISA and VSSA infections were similar (OR, 1.18; 95\% CI, 0.81-1.74). ${ }^{18}$ However, 190 these findings may be limited by the variability in definitions used and the predominately 191 retrospective designs of the original studies. While the lack of association between hVISA and 192 mortality can be partly explained by strain characteristics (e.g., decreased virulence) and host 
193 immune responses, sufficiently sized studies are needed to accurately determine if such an 194 association exits. ${ }^{47}$

196 Infections caused by VISA may also lead to recurrent infections, prolonged fevers and bacteremia, 197 vancomycin treatment failure, and increased hospital stay., ${ }^{72,33,44}$ In a single-center, retrospective 198 study, 6 patients with VISA had a significantly longer duration of bacteremia compared to 22 with 199 hVISA $(12.1 \pm 13.1$ days vs. $3.3 \pm 3.9$ days, respectively; $\mathrm{P}=0.001) .{ }^{43}$ Significant differences in 200 mortality between VISA and hVISA were not observed. However, rates of attributable mortality 201 between hVISA and VSSA ( $\mathrm{n}=215)$ were similar (9.1\% vs. $8.4 \%$, respectively) while those 202 between VISA and VSSA (33.3\% vs. 8.4\%) were not. ${ }^{43}$ Although this study had several 203 limitations including a small sample size and bias through selective inclusion of isolates, the 204 findings suggest that VISA may have more severe clinical implications and impact on patient 205 outcomes. To date, no other published study has evaluated the outcomes of VISA infections, 206 possibly because of the rarity of VISA infections.

\section{Treatment Options for hVISA/VISA Infections}

209 Although reports of vancomycin failure have emerged, no data demonstrate superior outcomes 210 with alternative antimicrobials. Alternative antimicrobial agents with activity against 211 hVISA/VISA include daptomycin, linezolid, ceftaroline, trimethoprim/sulfamethoxazole, 
212 tigecycline, quinupristin/dalfopristin, and the combination of vancomycin or daptomycin with a 213 beta-lactam. ${ }^{12}$

\section{Daptomycin}

216 Daptomycin is a potential treatment option for hVISA and VISA infections and, although it does

217 have activity against MRSA, previous vancomycin exposure can result in some degree of cross-

218 resistance to daptomycin. ${ }^{48,49}$ Several studies have noted an in vitro association between

219 increasing vancomycin MICs and increasing daptomycin non-susceptibility. ${ }^{48-50}$ The highest rate

220 of daptomycin non-susceptibility was reported in a study evaluating 47 Australian hVISA and

221 VISA isolates never exposed to daptomycin. ${ }^{50}$ The investigators noted daptomycin non-

222 susceptibly in $15 \%$ of hVISA and $38 \%$ of VISA strains. ${ }^{50}$ Because bactericidal activity with

223 daptomycin is concentration dependent, higher doses may be necessary to treat hVISA and VISA

224 infections with elevated daptomycin MICs, high inoculum infections (e.g., endocarditis), and

225 infection sites characterized by poor antimicrobial penetration. ${ }^{51}$ High-dose daptomycin may

226 prevent the selection or development of isolates with reduced susceptibility to daptomycin and

227 subsequent treatment failure. ${ }^{51}$

229 An in vitro study observed more rapid reduction of bacterial burden of hVISA and VISA in 230 simulated endocardial vegetations with high-dose daptomycin (10 mg/kg/day for 8 days $)$ and dose

231 de-escalation (10 mg/kg/day for 4 days followed by $6 \mathrm{mg} / \mathrm{kg} /$ day for 4 days) regimens compared 232 to that of the standard (6 mg/kg/day for 8 days) and dose escalation $(6 \mathrm{mg} / \mathrm{kg} / \mathrm{day}$ for 4 days 233 followed by $10 \mathrm{mg} / \mathrm{kg} /$ day for 4 days) regimens. ${ }^{51}$ With respect to hVISA, the dose de-escalation 234 regimen had a significantly increased killing effect on the hVISA strain compared to the dose 
235 escalation regimen $(\mathrm{P}<0.024) .{ }^{51}$ The investigators concluded that these daptomycin dosing 236 approaches may lead to a faster cure of bacteremia in vivo and prevent the emergence of 237 daptomycin non-susceptibility. ${ }^{51}$ However, no in vivo studies evaluating de-escalation dosing and 238 the appropriate duration of high-dose daptomycin have been published. The role of high-dose 239 daptomycin alone in patients with hVISA or VISA infections is unclear. Until more evidence is 240 available, caution is required when considering daptomycin in patients who may be at risk for 241 hVISA or VISA infections (e.g. high-bacterial load infections, vancomycin failure). The 242 determination of daptomycin susceptibility in these patients may also guide therapeutic decision 243 making.

245 Linezolid

246 The role of linezolid for the treatment of invasive hVISA and VISA infections is also in question.

247 Successful use of linezolid alone or in combination with other antimicrobial agents has been 248 described in several case reports of vancomycin heteroresistant and intermediate MRSA 249 endocarditis and bacteremias after vancomycin failure and in some cases after daptomycin 250 failure..$^{8,52-55}$ In one case report, a 60 year old male with an automatic implantable cardioverter251 defibrillator (AICD) presented with bacteremia and endocarditis initially caused by MRSA which 252 later developed into hVISA, then daptomycin non-susceptible VISA after exposure to vancomycin 253 and daptomycin. ${ }^{55}$ The patient initially received 6 weeks of vancomycin (trough concentrations 254 between $\geq 15 \mu \mathrm{g} / \mathrm{mL}$ and $\leq 21 \mu \mathrm{g} / \mathrm{mL}$ ), followed by approximately 25 days of daptomycin (6 $255 \mathrm{mg} / \mathrm{kg}$ every 48 hours, renal dose adjusted). During therapy with daptomycin the defibrillator 256 generator and leads were removed however, the patient was persistently bacteremic and febrile. 257 Blood cultures cleared after therapy was switched to linezolid and trimethoprim/sulfamethoxazole. 
258 The patient received at least 28 days of the combination and 6 weeks of linezolid monotherapy in 259 total since the last positive blood culture. One year post-treatment the patient had no infection

260 recurrence. After failing vancomycin and daptomycin therapy, this patient's VISA infection was

261 successfully treated with linezolid. While other case reports have shown similar outcomes with

262 the use of linezolid, in vitro studies have not shown the same efficacy. ${ }^{56}$ Evidence to recommend

263 the use of linezolid for hVISA and VISA is insufficient. Further study is needed to evaluate 264 linezolid alone or in combination for hVISA and VISA infections.

266 Ceftaroline

267 Ceftaroline has potent in vitro bactericidal activity against MRSA including hVISA, VISA, and 268 daptomycin non-susceptible (DNS) MRSA strains. ${ }^{57}$ The use of ceftaroline in the treatment of 269 invasive infections (e.g., endocarditis, bacteremia, osteomyelitis) caused by hVISA, VISA, and 270 DNS MRSA is supported by data from in vivo animal studies and human case reports. ${ }^{58-61}$ In a 271 recent case series report, a patient with DNS VISA bacteremia and endocarditis was successfully 272 treated with 6 weeks of ceftaroline. The patient initially received and failed vancomycin therapy. ${ }^{62}$ 273 Blood cultures cleared within 48 hours of switching to daptomycin $(6 \mathrm{mg} / \mathrm{kg} / \mathrm{day})$. However, 274 subsequent blood cultures were positive and revealed DNS VISA. Daptomycin was discontinued, 275 and ceftaroline (600 mg IV every 8 hours) was initiated. While on ceftaroline, blood cultures 276 cleared within 48 hours and remained sterile. In vitro pharmacokinetic/pharmacodynamic studies 277 reported enhanced ceftaroline activity against hVISA, VISA, and DNS MRSA as vancomycin and 278 daptomycin susceptibilities decreased, which have been referred to as the "seesaw effect". ${ }^{58-60}$ 279 While further study is needed, ceftaroline appears to be a safe and effective alternative in the 
280 treatment of invasive hVISA, VISA, and DNS MRSA infections given its bactericidal activity,

281 favorable safety profile, and emerging data.

282

283 Combination therapy

284 The combination of vancomycin or daptomycin and a beta-lactam antimicrobial has also been

285 studied for treatment of hVISA and VISA infections. Beta-lactams that have been evaluated for

286 synergistic activity with vancomycin or daptomycin include ceftaroline, cefazolin, and

287 piperacillin-tazobactam. ${ }^{63-66}$ In vitro and clinical case report data evaluating the combination of 288 high-dose daptomycin ( $10 \mathrm{mg} / \mathrm{kg} / \mathrm{day})$ and trimethoprim/sulfamethoxazole also appear promising 289 for the treatment of hVISA, VISA, and DNS MRSA infections. ${ }^{67,68}$ In vitro studies have 290 demonstrated improved kill rates with these antimicrobial combinations. ${ }^{63-65}$ Investigators 291 hypothesize that beta-lactam exposure may influence vancomycin-cell wall interactions to 292 improve vancomycin activity, although further investigation is warranted. ${ }^{63}$ In summary, 293 preliminary experimental studies show possible prospects for the treatment of hVISA and VISA 294 infections. However, it is not yet clear which treatment options correlate with optimal clinical 295 outcomes for patients with confirmed hVISA or VISA infections.

297 Infection Control: Preventing the Dissemination of hVISA/VISA

298 As with MRSA, hVISA and VISA can colonize humans and the environment despite eradication 299 efforts. The CDC has made several recommendations in an attempt to prevent the emergence of 300 vancomycin non-susceptible infections. ${ }^{42}$ Infections with confirmed VISA should be reported to 301 infection-control personnel, the patient's primary caregiver, medical ward staff, local and state 302 departments of health, and the CDC. Patients and their caregivers should be educated regarding 
303 wound care, physical hygiene, and signs of infection. ${ }^{69}$ Contact isolation in both the inpatient and

304 outpatient setting may also limit further emergence. Adherence to recommended infection

305 prevention and control guidelines, appropriate antibiotic prescribing through antimicrobial

306 stewardship programs, and active surveillance in a cohesive health care system are essential to

307 prevent further emergence of hVISA and VISA colonization and infection.

309 Conclusions

310 The evolution of S. aureus to MRSA and now to hVISA and VISA is an important and ongoing

311 public health concern. Vancomycin is the drug of choice for invasive MRSA infections, however,

312 its use is under question. Over-use, suboptimal concentrations, or inappropriate use of vancomycin

313 is speculated to be a major contributor in the emergence of hVISA and VISA. Most alarming are

314 the poor outcomes that have been associated with hVISA and VISA infections and the limited

315 antimicrobials available to treat these infections. Proper detection methods are necessary for

316 accurate surveillance, guidance on therapeutic decision-making, and a full understanding of the

317 implications of hVISA/VISA infections. Until then, patients who are at risk for hVISA/VISA

318 infections and failing vancomycin therapy may warrant further confirmatory testing for

319 hVISA/VISA. Based on currently available data, clinicians should, with vigilance, continue to use

320 vancomycin per the Infectious Diseases Society of America guidelines. ${ }^{2,3}$ Alternative therapies

321 should be considered in patients with risk factors for hVISA/VISA who are not responding 322 clinically to vancomycin despite source control and a vancomycin MIC $\leq 2 \mu \mathrm{g} / \mathrm{mL}$. In patients 323 infected with VISA (vancomycin MIC $4-8 \mu \mathrm{g} / \mathrm{mL}$ ), an alternative antimicrobial should be 324 considered. Caution is advised when deciding to use daptomycin in patients with hVISA/VISA 
325 infections because of the potential for cross-resistance. To prevent further resistance, appropriate

326 use of antimicrobials and implementation of infection-control guidelines are imperative. 


\section{REFERENCES}

1. Dantes $\mathrm{R}, \mathrm{Mu} \mathrm{Y}$, Belflower $\mathrm{R}$, et al. National burden of invasive methicillinresistant Staphylococcus aureus infections, United States, 2011. JAMA internal medicine. Nov 25 2013;173(21):1970-1978.

2. Liu C, Bayer A, Cosgrove SE, et al. Clinical practice guidelines by the infectious diseases society of america for the treatment of methicillin-resistant Staphylococcus aureus infections in adults and children. Clinical infectious diseases : an official publication of the Infectious Diseases Society of America. Feb $12011 ; 52(3): \mathrm{e} 18-55$.

3. Rybak M, Lomaestro B, Rotschafer JC, et al. Therapeutic monitoring of vancomycin in adult patients: a consensus review of the American Society of Health-System Pharmacists, the Infectious Diseases Society of America, and the Society of Infectious Diseases Pharmacists. American journal of health-system pharmacy : AJHP : official journal of the American Society of Health-System Pharmacists. Jan 1 2009;66(1):82-98.

4. Jones RN. Microbiological features of vancomycin in the 21st century: minimum inhibitory concentration creep, bactericidal/static activity, and applied breakpoints to predict clinical outcomes or detect resistant strains. Clinical infectious diseases : an official publication of the Infectious Diseases Society of America. Jan 1 2006;42 Suppl 1:S13-24.

5. Hiramatsu K. Vancomycin-resistant Staphylococcus aureus: a new model of antibiotic resistance. The Lancet. Infectious diseases. Oct 2001;1(3):147-155.

6. Casapao AM, Leonard SN, Davis SL, et al. Clinical outcomes in patients with heterogeneous vancomycin-intermediate Staphylococcus aureus (hVISA) bloodstream infection. Antimicrob Agents Chemother. Jun 242013.

7. Charles PG, Ward PB, Johnson PD, Howden BP, Grayson ML. Clinical features associated with bacteremia due to heterogeneous vancomycin-intermediate Staphylococcus aureus. Clinical infectious diseases : an official publication of the Infectious Diseases Society of America. Feb 1 2004;38(3):448-451.

8. Howden BP, Ward PB, Charles PG, et al. Treatment outcomes for serious infections caused by methicillin-resistant Staphylococcus aureus with reduced vancomycin susceptibility. Clinical infectious diseases : an official publication of the Infectious Diseases Society of America. Feb 15 2004;38(4):521-528. 
9. Hiramatsu K, Aritaka N, Hanaki H, et al. Dissemination in Japanese hospitals of strains of Staphylococcus aureus heterogeneously resistant to vancomycin. Lancet. Dec 6 1997;350(9092):1670-1673.

10. Hiramatsu K, Hanaki H, Ino T, Yabuta K, Oguri T, Tenover FC. Methicillinresistant Staphylococcus aureus clinical strain with reduced vancomycin susceptibility. J Antimicrob Chemother. Jul 1997;40(1):135-136.

11. Howden BP, Davies JK, Johnson PD, Stinear TP, Grayson ML. Reduced vancomycin susceptibility in Staphylococcus aureus, including vancomycinintermediate and heterogeneous vancomycin-intermediate strains: resistance mechanisms, laboratory detection, and clinical implications. Clinical microbiology reviews. Jan 2010;23(1):99-139.

12. Horne KC, Howden BP, Grabsch EA, et al. Prospective comparison of the clinical impacts of heterogeneous vancomycin-intermediate methicillin-resistant Staphylococcus aureus (MRSA) and vancomycin-susceptible MRSA. Antimicrob Agents Chemother. Aug 2009;53(8):3447-3452.

13. Leonard SN, Rybak MJ. Evaluation of vancomycin and daptomycin against methicillin-resistant Staphylococcus aureus and heterogeneously vancomycinintermediate $\mathrm{S}$. aureus in an in vitro pharmacokinetic/pharmacodynamic model with simulated endocardial vegetations. $J$ Antimicrob Chemother. Jan 2009;63(1):155-160.

14. Maor Y, Hagin M, Belausov N, Keller N, Ben-David D, Rahav G. Clinical features of heteroresistant vancomycin-intermediate Staphylococcus aureus bacteremia versus those of methicillin-resistant S. aureus bacteremia. J Infect Dis. Mar 1 2009;199(5):619-624.

15. Musta AC, Riederer K, Shemes S, et al. Vancomycin MIC plus heteroresistance and outcome of methicillin-resistant Staphylococcus aureus bacteremia: trends over 11 years. J Clin Microbiol. Jun 2009;47(6):1640-1644.

16. Rybak MJ, Leonard SN, Rossi KL, Cheung CM, Sader HS, Jones RN. Characterization of vancomycin-heteroresistant Staphylococcus aureus from the metropolitan area of Detroit, Michigan, over a 22-year period (1986 to 2007). J Clin Microbiol. Sep 2008;46(9):2950-2954.

17. Satola SW, Farley MM, Anderson KF, Patel JB. Comparison of detection methods for heteroresistant vancomycin-intermediate Staphylococcus aureus, with the population analysis profile method as the reference method. J Clin Microbiol. Jan 2011;49(1):177-183. 
18. van Hal SJ, Paterson DL. Systematic review and meta-analysis of the significance of heterogeneous vancomycin-intermediate Staphylococcus aureus isolates. Antimicrob Agents Chemother. Jan 2011;55(1):405-410.

19. van Hal SJ, Wehrhahn MC, Barbagiannakos T, et al. Performance of various testing methodologies for detection of heteroresistant vancomycin-intermediate Staphylococcus aureus in bloodstream isolates. $J$ Clin Microbiol. Apr 2011;49(4):1489-1494.

20. Sancak B, Ercis S, Menemenlioglu D, Colakoglu S, Hascelik G. Methicillinresistant Staphylococcus aureus heterogeneously resistant to vancomycin in a Turkish university hospital. J Antimicrob Chemother. Sep 2005;56(3):519-523.

21. Boyle-Vavra S, Berke SK, Lee JC, Daum RS. Reversion of the glycopeptide resistance phenotype in Staphylococcus aureus clinical isolates. Antimicrob Agents Chemother. Feb 2000;44(2):272-277.

22. Richter SS, Diekema DJ, Heilmann KP, et al. Activities of vancomycin, ceftaroline, and mupirocin against Staphylococcus aureus isolates collected in a 2011 national surveillance study in the United States. Antimicrob Agents Chemother. 2014;58(2):740-745.

23. Richter SS, Satola SW, Crispell EK, et al. Detection of Staphylococcus aureus isolates with heterogeneous intermediate-level resistance to vancomycin in the United States. J Clin Microbiol. Dec 2011;49(12):4203-4207.

24. Clinical and Laboratory Standards Institute. Performance Standards for Antimicrobial Susceptibility Testing. Sixteenth international supplement. M100S16. Wayne, PA: CLSI, 2006.

25. Tenover FC, Moellering RC, Jr. The rationale for revising the Clinical and Laboratory Standards Institute vancomycin minimal inhibitory concentration interpretive criteria for Staphylococcus aureus. Clinical infectious diseases : an official publication of the Infectious Diseases Society of America. May 1 2007;44(9):1208-1215.

26. Wootton M, Howe RA, Hillman R, Walsh TR, Bennett PM, MacGowan AP. A modified population analysis profile (PAP) method to detect hetero-resistance to vancomycin in Staphylococcus aureus in a UK hospital. J Antimicrob Chemother. Apr 2001;47(4):399-403.

27. Leonard SN, Rossi KL, Newton KL, Rybak MJ. Evaluation of the Etest GRD for the detection of Staphylococcus aureus with reduced susceptibility to glycopeptides. J Antimicrob Chemother. Mar 2009;63(3):489-492. 
28. Wootton M, MacGowan AP, Walsh TR, Howe RA. A multicenter study evaluating the current strategies for isolating Staphylococcus aureus strains with reduced susceptibility to glycopeptides. J Clin Microbiol. Feb 2007;45(2):329-332.

29. Yusof A, Engelhardt A, Karlsson A, et al. Evaluation of a new Etest vancomycinteicoplanin strip for detection of glycopeptide-intermediate Staphylococcus aureus (GISA), in particular, heterogeneous GISA. $J$ Clin Microbiol. Sep 2008;46(9):3042-3047.

30. Centers for Disease Control and Prevention. Laboratory Detection of VancomycinIntermediate/Resistant Staphylococcus aureus (VISA/VRSA). http://www.cdc.gov/ncidod/dhqp/ar_visavrsa_labFAQ.html\#1. Accessed August 4, 2014.

31. Rybak MJ, Vidaillac C, Sader HS, et al. Evaluation of vancomycin susceptibility testing for methicillin-resistant Staphylococcus aureus: comparison of Etest and three automated testing methods. J Clin Microbiol. Jul 2013;51(7):2077-2081.

32. van Hal SJ, Lodise TP, Paterson DL. The clinical significance of vancomycin minimum inhibitory concentration in Staphylococcus aureus infections: a systematic review and meta-analysis. Clinical infectious diseases : an official publication of the Infectious Diseases Society of America. Mar 2012;54(6):755771.

33. Howden BP, Johnson PD, Ward PB, Stinear TP, Davies JK. Isolates with low-level vancomycin resistance associated with persistent methicillin-resistant Staphylococcus aureus bacteremia. Antimicrob Agents Chemother. Sep 2006;50(9):3039-3047.

34. Cui L, Ma X, Sato K, et al. Cell wall thickening is a common feature of vancomycin resistance in Staphylococcus aureus. J Clin Microbiol. Jan 2003;41(1):5-14.

35. Pfeltz RF, Singh VK, Schmidt JL, et al. Characterization of passage-selected vancomycin-resistant Staphylococcus aureus strains of diverse parental backgrounds. Antimicrob Agents Chemother. Feb 2000;44(2):294-303.

36. Sakoulas G, Eliopoulos GM, Fowler VG, Jr., et al. Reduced susceptibility of Staphylococcus aureus to vancomycin and platelet microbicidal protein correlates with defective autolysis and loss of accessory gene regulator (agr) function. Antimicrob Agents Chemother. Jul 2005;49(7):2687-2692.

37. Sakoulas G, Eliopoulos GM, Moellering RC, Jr., et al. Accessory gene regulator (agr) locus in geographically diverse Staphylococcus aureus isolates with reduced susceptibility to vancomycin. Antimicrob Agents Chemother. May 2002;46(5):1492-1502. 
38. Hidayat LK, Hsu DI, Quist R, Shriner KA, Wong-Beringer A. High-dose vancomycin therapy for methicillin-resistant Staphylococcus aureus infections: efficacy and toxicity. Arch Intern Med. Oct 23 2006;166(19):2138-2144.

39. Lodise TP, Graves J, Evans A, et al. Relationship between vancomycin MIC and failure among patients with methicillin-resistant Staphylococcus aureus bacteremia treated with vancomycin. Antimicrob Agents Chemother. Sep 2008;52(9):33153320.

40. Jacob JT, DiazGranados CA. High vancomycin minimum inhibitory concentration and clinical outcomes in adults with methicillin-resistant Staphylococcus aureus infections: a meta-analysis. International journal of infectious diseases : IJID : official publication of the International Society for Infectious Diseases. Feb 2013;17(2):e93-e100.

41. Chen H, Liu Y, Sun W, Chen M, Wang H. The incidence of heterogeneous vancomycin-intermediate Staphylococcus aureus correlated with increase of vancomycin MIC. Diagnostic microbiology and infectious disease. Nov 2011;71(3):301-303.

42. Rose WE, Leonard SN, Rossi KL, Kaatz GW, Rybak MJ. Impact of inoculum size and heterogeneous vancomycin-intermediate Staphylococcus aureus (hVISA) on vancomycin activity and emergence of VISA in an in vitro pharmacodynamic model. Antimicrob Agents Chemother. Feb 2009;53(2):805-807.

43. Khatib R, Jose J, Musta A, et al. Relevance of vancomycin-intermediate susceptibility and heteroresistance in methicillin-resistant Staphylococcus aureus bacteraemia. J Antimicrob Chemother. Jul 2011;66(7):1594-1599.

44. Fong RK, Low J, Koh TH, Kurup A. Clinical features and treatment outcomes of vancomycin-intermediate Staphylococcus aureus (VISA) and heteroresistant vancomycin-intermediate Staphylococcus aureus (hVISA) in a tertiary care institution in Singapore. European journal of clinical microbiology \& infectious diseases : official publication of the European Society of Clinical Microbiology. Aug 2009;28(8):983-987.

45. Fridkin SK, Hageman J, McDougal LK, et al. Epidemiological and microbiological characterization of infections caused by Staphylococcus aureus with reduced susceptibility to vancomycin, United States, 1997-2001. Clinical infectious diseases : an official publication of the Infectious Diseases Society of America. Feb 15 2003;36(4):429-439.

46. Satola SW, Lessa FC, Ray SM, et al. Clinical and laboratory characteristics of invasive infections due to methicillin-resistant Staphylococcus aureus isolates demonstrating a vancomycin MIC of 2 micrograms per milliliter: lack of effect of 
heteroresistant vancomycin-intermediate S. aureus phenotype. J Clin Microbiol. Apr 2011;49(4):1583-1587.

47. Howden BP, Smith DJ, Mansell A, et al. Different bacterial gene expression patterns and attenuated host immune responses are associated with the evolution of low-level vancomycin resistance during persistent methicillin-resistant Staphylococcus aureus bacteraemia. BMC microbiology. 2008;8:39.

48. Sakoulas G, Alder J, Thauvin-Eliopoulos C, Moellering RC, Jr., Eliopoulos GM. Induction of daptomycin heterogeneous susceptibility in Staphylococcus aureus by exposure to vancomycin. Antimicrob Agents Chemother. Apr 2006;50(4):15811585.

49. Patel JB, Jevitt LA, Hageman J, McDonald LC, Tenover FC. An association between reduced susceptibility to daptomycin and reduced susceptibility to vancomycin in Staphylococcus aureus. Clinical infectious diseases : an official publication of the Infectious Diseases Society of America. Jun 1 2006;42(11):16521653.

50. Kelley PG, Gao W, Ward PB, Howden BP. Daptomycin non-susceptibility in vancomycin-intermediate Staphylococcus aureus (VISA) and heterogeneous-VISA (hVISA): implications for therapy after vancomycin treatment failure. J Antimicrob Chemother. May 2011;66(5):1057-1060.

51. Vidaillac C, Steed ME, Rybak MJ. Impact of dose de-escalation and escalation on daptomycin's pharmacodynamics against clinical methicillin-resistant Staphylococcus aureus isolates in an in vitro model. Antimicrob Agents Chemother. May 2011;55(5):2160-2165.

52. Andrade-Baiocchi S, Tognim MC, Baiocchi OC, Sader HS. Endocarditis due to glycopeptide-intermediate Staphylococcus aureus: case report and strain characterization. Diagnostic microbiology and infectious disease. Feb 2003;45(2):149-152.

53. Huang YT, Hsiao $\mathrm{CH}$, Liao $\mathrm{CH}$, Lee $\mathrm{CW}$, Hsueh PR. Bacteremia and infective endocarditis caused by a non-daptomycin-susceptible, vancomycin-intermediate, and methicillin-resistant Staphylococcus aureus strain in Taiwan. J Clin Microbiol. Mar 2008;46(3):1132-1136.

54. Leung KT, Tong MK, Siu YP, Lam CS, Ng HL, Lee HK. Treatment of vancomycin-intermediate Staphylcoccus aureus endocarditis with linezolid. Scandinavian journal of infectious diseases. 2004;36(6-7):483-485.

55. Tenover FC, Sinner SW, Segal RE, et al. Characterisation of a Staphylococcus aureus strain with progressive loss of susceptibility to vancomycin and daptomycin 
during therapy. International journal of antimicrobial agents. Jun 2009;33(6):564568.

56. Leonard SN, Szeto YG, Zolotarev M, Grigoryan IV. Comparative in vitro activity of telavancin, vancomycin and linezolid against heterogeneously vancomycinintermediate Staphylococcus aureus (hVISA). International journal of antimicrobial agents. Jun 2011;37(6):558-561.

57. Saravolatz L, Pawlak J, Johnson L. In vitro activity of ceftaroline against community-associated methicillin-resistant, vancomycin-intermediate, vancomycin-resistant, and daptomycin-nonsusceptible Staphylococcus aureus isolates. Antimicrob Agents Chemother. Jul 2010;54(7):3027-3030.

58. Steed M, Vidaillac C, Rybak MJ. Evaluation of ceftaroline activity versus daptomycin (DAP) against DAP-nonsusceptible methicillin-resistant Staphylococcus aureus strains in an in vitro pharmacokinetic/pharmacodynamic model. Antimicrob Agents Chemother. Jul 2011;55(7):3522-3526.

59. Vidaillac C, Leonard SN, Rybak MJ. In vitro activity of ceftaroline against methicillin-resistant Staphylococcus aureus and heterogeneous vancomycinintermediate S. aureus in a hollow fiber model. Antimicrob Agents Chemother. Nov 2009;53(11):4712-4717.

60. Werth BJ, Steed ME, Kaatz GW, Rybak MJ. Evaluation of ceftaroline activity against heteroresistant vancomycin-intermediate Staphylococcus aureus and vancomycin-intermediate methicillin-resistant $S$. aureus strains in an in vitro pharmacokinetic/pharmacodynamic model: exploring the "seesaw effect". Antimicrob Agents Chemother. Jun 2013;57(6):2664-2668.

61. Jacqueline C, Caillon J, Le Mabecque V, et al. In vivo efficacy of ceftaroline (PPI0903), a new broad-spectrum cephalosporin, compared with linezolid and vancomycin against methicillin-resistant and vancomycin-intermediate Staphylococcus aureus in a rabbit endocarditis model. Antimicrob Agents Chemother. Sep 2007;51(9):3397-3400.

62. Ho TT, Cadena J, Childs LM, Gonzalez-Velez M, Lewis JS, 2nd. Methicillinresistant Staphylococcus aureus bacteraemia and endocarditis treated with ceftaroline salvage therapy. J Antimicrob Chemother. May 2012;67(5):1267-1270.

63. Werth BJ, Vidaillac C, Murray KP, et al. Novel combinations of vancomycin plus ceftaroline or oxacillin against methicillin-resistant vancomycin-intermediate Staphylococcus aureus (VISA) and heterogeneous VISA. Antimicrob Agents Chemother. May 2013;57(5):2376-2379. 
64. Hagihara M, Wiskirchen DE, Kuti JL, Nicolau DP. In vitro pharmacodynamics of vancomycin and cefazolin alone and in combination against methicillin-resistant Staphylococcus aureus. Antimicrob Agents Chemother. Jan 2012;56(1):202-207.

65. Dilworth TJ, Sliwinski J, Ryan K, Dodd M, Mercier RC. Evaluation of vancomycin in combination with piperacillin-tazobactam or oxacillin against clinical methicillin-resistant Staphylococcus aureus isolates and vancomycin-intermediate Staphylococcus aureus isolates in vitro. Antimicrob Agents Chemother. Nov 25 2013.

66. Werth BJ, Barber KE, Ireland CE, Rybak MJ. Evaluation of ceftaroline, vancomycin, daptomycin, or ceftaroline plus daptomycin against daptomycinnonsusceptible methicillin-resistant Staphylococcus aureus in an in vitro pharmacokinetic/pharmacodynamic model of simulated endocardial vegetations. Antimicrob Agents Chemother. Jun 2014;58(6):3177-3181.

67. Avery LM, Steed ME, Woodruff AE, Hasan M, Rybak MJ. Daptomycinnonsusceptible vancomycin-intermediate staphylococcus aureus vertebral osteomyelitis cases complicated by bacteremia treated with high-dose daptomycin and trimethoprim-sulfamethoxazole. Antimicrob Agents Chemother. Nov 2012;56(11):5990-5993.

68. Steed ME, Werth BJ, Ireland CE, Rybak MJ. Evaluation of the novel combination of high-dose daptomycin plus trimethoprim-sulfamethoxazole against daptomycinnonsusceptible methicillin-resistant Staphylococcus aureus using an in vitro pharmacokinetic/pharmacodynamic model of simulated endocardial vegetations. Antimicrob Agents Chemother. Nov 2012;56(11):5709-5714.

69. CDC Reminds Clinical Laboratories and Healthcare Infection Preventionists of their Role in the Search and Containment of Vancomycin-Resistant Staphylococcus aureus (VRSA). 2010 http://www.cdc.gov/HAI/settings/lab/vrsa_lab_search_containment.html. Accessed August 4, 2014.

70. Liu C, Chambers HF. Staphylococcus aureus with heterogeneous resistance to vancomycin: epidemiology, clinical significance, and critical assessment of diagnostic methods. Antimicrob Agents Chemother. Oct 2003;47(10):3040-3045. 
Table 1. CLSI susceptibility definitions for vancomycin ${ }^{24,25}$

\begin{tabular}{|c|c|c|}
\hline & $\begin{array}{c}\text { 2006 CLSI Update } \\
\text { MIC }\end{array}$ & $\begin{array}{c}\text { Previous CLSI Breakpoints } \\
\text { MIC }\end{array}$ \\
\hline VSSA & $\leq 2 \mu \mathrm{g} / \mathrm{mL}^{\mathrm{a}}$ & $\leq 4 \mu \mathrm{g} / \mathrm{mL}$ \\
\hline VISA & $4-8 \mu \mathrm{g} / \mathrm{mL}$ & $8-16 \mu \mathrm{g} / \mathrm{mL}$ \\
\hline VRSA & $\geq 16 \mu \mathrm{g} / \mathrm{mL}$ & $\geq 32 \mu \mathrm{g} / \mathrm{mL}$ \\
\hline
\end{tabular}

CLSI = Clinical and Laboratory Standards Institute; MIC = minimum inhibitory concentration;

VISA = vancomycin intermediate $S$. aureus; VRSA = vancomycin resistant $S$. aureus; VSSA = vancomycin susceptible $S$. aureus;

${ }^{a}$ May contain heteroresistant intermediate susceptible subpopulations with MIC $>4 \mu \mathrm{g} / \mathrm{mL}$. Heteroresistant vancomycin intermediate $S$. aureus (hVISA) isolates are not identified by CLSI and can occur at vancomycin MICs as low as $0.5 \mu \mathrm{g} / \mathrm{mL}$. 
Table 2. Advantages and disadvantages of laboratory detection methods for hVISA

\begin{tabular}{|c|c|c|}
\hline \multicolumn{3}{|c|}{ Confirmatory Methods } \\
\hline Method & Advantages & Disadvantages \\
\hline $\mathrm{PAP}^{4,11,13,26,70}$ & $\begin{array}{l}\text { - Considered the "gold } \\
\text { standard" } \\
\text { - High reproducibility and } \\
\text { accurate detection } \\
\text { - Definitive confirmation: } \\
\text { Modified PAP }\end{array}$ & $\begin{array}{l}\text { - No data to show superiority to } \\
\text { other techniques } \\
\text { - High labor intensity } \\
\text { - High-cost } \\
\text { - Long turn-around time }\end{array}$ \\
\hline \multicolumn{3}{|c|}{ Screening Methods } \\
\hline Method & Advantages & Disadvantages \\
\hline $\begin{array}{l}\text { GRD E-test } \\
\text { (AB Biodisk) }^{17,19,27}\end{array}$ & $\begin{array}{l}\text { - Results ready to read following } \\
24 \text { hours of incubation } \\
\text { - Uses standard bacterial } \\
\text { inoculum }\end{array}$ & \begin{tabular}{l|l} 
- Unreliable specificity and \\
sensitivity
\end{tabular} \\
\hline $\begin{array}{l}\text { MET or } \\
\text { High inoculum } \\
\text { method }^{11,29}\end{array}$ & $\begin{array}{l}\text { - } 100 \% \text { reproducibility } \\
\text { - Easily performed }\end{array}$ & $\begin{array}{l}\text { - Testing performed on } \\
\text { nonstandard media while } \\
\text { utilizing a standard McFarland } \\
\text { suspension } \\
\text { - Results of MET are cut-off } \\
\text { points, not true MICs }\end{array}$ \\
\hline $\begin{array}{l}\text { BHI screen agar } \\
\text { plates } 7,17,28\end{array}$ & • Easily performed & $\begin{array}{l}\text { - Poor reproducibility } \\
\text { - Many variations; some studies } \\
\text { screened with a different agar, } \\
\text { inoculum size, or used } \\
\text { suspensions with higher } \\
\text { bacterial concentration }\end{array}$ \\
\hline
\end{tabular}


Table 3. Predictors and outcomes of hVISA and VISA

\begin{tabular}{|l|l|}
\hline \multicolumn{1}{|c|}{ Predictors } & \multicolumn{1}{c|}{ Outcomes } \\
\hline - Previous vancomycin use & • Long duration of bacteremia, days \\
- Prior MRSA infection or colonization & - Persistent fever \\
- High bacterial load infections ${ }^{\mathrm{a}}$ & - Recurrent infections \\
- Persistent bacteremia & - Vancomycin treatment failure \\
- Initally low serum vancomycin levels & - Prolonged hospitalization \\
- Presence of indwelling devices & \\
\hline
\end{tabular}

hVISA = heteroresistant vancomycin intermediate $S$. aureus $;$ MRSA = methicillin resistant $S$. aureus; VISA = vancomycin intermediate $S$. aureus ${ }^{\text {a }}$ E.g.bacteremia, endocarditis, osteomyelitis, deep abscess, or prosthetic joint infection 